\title{
Phase II trial of docetaxel in advanced or metastatic endometrial cancer: a Japanese Cooperative Study
}

\author{
N Katsumata*, , K Noda ${ }^{2}$, S Nozawa ${ }^{3}$, R Kitagawa', R Nishimura ${ }^{4}$, S Yamaguchi ${ }^{4}$, D Aoki ${ }^{3}$, N Susumu ${ }^{3}$,

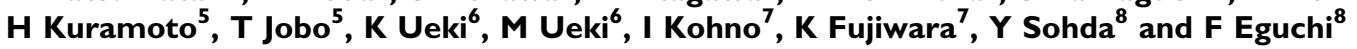 \\ 'Department of Medical Oncology, National Cancer Center Hospital, 104-0045 Tokyo, Japan; ${ }^{2}$ Kinki University, Osakasayama, Japan; ${ }^{3}$ Department of \\ Obstetrics and Gynecology, School of Medicine, Keio University, I 60-8582 Tokyo, Japan; ${ }^{4}$ Department of Gynecology, Hyogo Medical Center for Adults, \\ 673-8558 Akashi, Japan; ${ }^{5}$ Department of Obstetrics and Gynecology, Kitasato University, 228-8555 Sagamihara, Japan; ${ }^{6}$ Department of Obstetrics and \\ Gynecology, Osaka Medical College, 569-8686 Takatsuki, Japan; 'D Department of Obstetrics and Gynecology,Kawasaki Medical School, 70 I-0192 \\ Kurashiki, Japan; ${ }^{8}$ Department of Obstetrics and Gynecology, Aso lizuka Hospital, 820-8505 lizuka, Japan
}

The purpose of this study was to determine whether docetaxel has antitumour activity in patients with advanced or recurrent endometrial carcinoma. Chemotherapy-naïve or previously treated patients (one regimen) with histopathologically documented endometrial carcinoma and Eastern Cooperative Oncology Group performance status $\leqslant 2$ entered the study. Docetaxel $70 \mathrm{mg} \mathrm{m}^{-2}$ was administered intravenously on day I of a 3-week cycle up to a maximum of six cycles. If patients responded well to docetaxel, additional cycles were administered until progressive disease or unacceptable toxicity occurred. Of 33 patients with a median age of 59 years (range, 39-74 years) who entered the study, 14 patients (42\%) had received one prior chemotherapy regimen. In all, 32 patients were evaluable for efficacy, yielding an overall response rate of 31\% (95\% confidence interval, 16.1-50.0\%); complete response and partial response (PR) were 3 and 28\%, respectively. Of 13 pretreated patients, three (23\%) had a PR. The median duration of response was 1.8 months. The median time to progression was 3.9 months. The predominant toxicity was grade $3-4$ neutropenia, occurring in $94 \%$ of the patients, although febrile neutropenia arose in $9 \%$ of the patients. Oedema was mild and infrequent. Docetaxel has antitumour activity in patients with advanced or recurrent endometrial carcinoma, including those previously treated with chemotherapy; however, the effect was transient and accompanied by pronounced neutropenia in most patients.

British Journal of Cancer (2005) 93, 999-1004. doi:10.1038/sj.bjc.66028I7 www.bjcancer.com

Published online 18 October 2005

(c) 2005 Cancer Research UK

Keywords: docetaxel; endometrial cancer; phase ॥

Most patients with endometrial cancer are diagnosed at an early stage when surgery alone may result in cure. However, the outcome for women with advanced stage or recurrent disease is poor and rarely curable. Both single-agent and combination regimens of chemotherapy have been studied in women with advanced endometrial carcinoma. Currently, no standard chemotherapy regimen for endometrial cancer exists, but single-agent doxorubicin is active, with responses observed in up to one-third of previously untreated patients (Moore et al, 1991). Other single agents with modest activity include cisplatin (Thigpen et al, 1984a, 1989) and carboplatin (van Wijk et al, 2003). Although the response rates with the combination doxorubicin-cisplatin appear to be higher than those achieved with either agent alone, there is no evidence that survival is any longer with combination therapy. In the Gynecologic Oncology Group (GOG) trial comparing doxorubicin alone with doxorubicin-cisplatin, the response rates and progression-free survival were better with the combination regimen (42 vs 25\%, 5.7 vs 3.8 months, respectively), but overall survival (OS) had not significantly improved (Thigpen et al, 2004).

*Correspondence: Dr N Katsumata; E-mail: nkatsuma@ncc.go.jp Received 22 July 2005; revised 19 September 2005; accepted 19 September 2005; published online 18 October 2005
The taxanes, paclitaxel and docetaxel, are potent chemotherapeutic agents that block tubulin depolymerisation, leading to the inhibition of microtubule dynamics, and have significant clinical efficacy for various solid tumours. Paclitaxel has been evaluated as an active agent for endometrial cancer (Ball et al, 1996; Lissoni et al, 1996; Lincoln et al, 2003). However, preclinical data show that docetaxel has increased potency and an improved therapeutic index compared with paclitaxel (Bissery et al, 1995), and its short 1-h infusion time offers a substantial clinical advantage over the prolonged infusion durations required with paclitaxel. Docetaxel and paclitaxel also have substantially different toxicity profiles. In particular, docetaxel has a significant lower incidence of neurotoxicity in comparison to paclitaxel (Hsu et al, 2004).

The present phase II trial was designed to evaluate the clinical efficacy and tolerability of docetaxel $70 \mathrm{mg} \mathrm{m}^{-2}$ in patients with advanced or recurrent endometrial cancer.

\section{PATIENTS AND METHODS}

\section{Eligibility criteria}

Eligible patients aged between 20 and 74 years, with a life expectancy in excess of 3 months, and Eastern Cooperative 
Oncology Group (ECOG) Performance Status (PS) of $0-2$ had histologically documented primary stage III, IV or recurrent endometrial carcinoma. Tumours were staged according to the International Federation of Gynecology and Obstetrics criteria. All patients had measurable disease according to the response evaluation criteria in solid tumours (RECIST) (Therasse et al, 2000). Measurable lesions defined unidimensionally were $\geqslant 20 \mathrm{~mm}$ using conventional imaging, or $\geqslant 10 \mathrm{~mm}$ with spiral computed tomographic scan. Patients were either chemotherapy-naive or had received one prior chemotherapy regimen for endometrial cancer, with 4 weeks between prior therapy and study treatment. Prior treatment with a taxane was not allowed. Adequate organ function was required for study entry: neutrophil count $\geqslant 2000 \mu \mathrm{l}^{-1}$, platelet count $\geqslant 100000 \mu \mathrm{l}^{-1}$, haemoglobin $\geqslant 9.0 \mathrm{~g} \mathrm{dl}^{-1}$, serum bilirubin level $\leqslant 1.5 \mathrm{mg} \mathrm{dl}^{-1}$, normal hepatic function (asparate aminotransferase (AST), alanine aminotransferase (ALT) and alkaline phosphatase (ALP) levels $\leqslant 2.5$ times upper limit of the institutional normal (ULN)), serum creatinine level $\leqslant 1.5 \mathrm{mg} \mathrm{dl}^{-1}, \mathrm{PaO}_{2}$ $\geqslant 60 \mathrm{mmHg}$ and normal electrocardiogram. Patients with any of the following conditions were excluded from the study: sarcoma component, active infection, severe heart disease, interstitial pneumonitis, past history of hypersensitivity, peripheral neuropathy, malignant or benign effusions requiring drainage, active brain metastasis, or active concomitant malignancy. All patients gave informed consent before entering this study, which was approved by the institutional review boards at all participating institutions.

\section{Treatment schedule}

Docetaxel $70 \mathrm{mg} \mathrm{m}^{-2}$ was infused over a 1-2-h period. The treatment was repeated every 3 weeks unless there was documented disease progression or unacceptable toxicity. Prophylactic medications for nausea, vomiting or hypersensitivity reactions were given if these symptoms occurred. No routine premedication was given for hypersensitivity reactions and fluid retention during the first cycle of treatment. The patient's physician identified all hypersensitivity reactions and, if deemed necessary, the investigator administered premedication drugs.

Treatment was delayed for up to 3 weeks in the event of toxicity, but was restarted when the neutrophil count was $\geqslant 1500 \mu \mathrm{l}^{-1}$, platelet count $\geqslant 100000 \mu \mathrm{l}^{-1}$, AST/ALT/ALP levels $\leqslant 2.5$ times ULN, and neuropathy or oedema $\leqslant$ grade 1 . Docetaxel dosage was reduced by $10 \mathrm{mg} \mathrm{m}^{-2}$ if febrile neutropenia occurred, if there was bleeding with grade 3-4 thrombocytopenia requiring a platelet transfusion, or if a patient experienced any grade 3-4 nonhaematologic toxicities except nausea, vomiting, anorexia, fatigue, alopecia or hypersensitivity.

\section{Response and toxicity evaluation}

The tumour response was assessed according to the standard RECIST criteria (Therasse et al, 2000). Target lesions included all measurable lesions up to a maximum of five lesions per organ and 10 lesions in total. Complete response (CR) was defined as the complete disappearance of all target and nontarget lesions, with no development of new disease. Partial response (PR) was defined as a reduction by $\geqslant 30 \%$ in the sum of the longest diameter of target lesions. Complete response or PRss were confirmed by repeat assessments performed no less than 4 weeks after the criteria for response were first met. Progressive disease (PD) was defined as an increase by $\geqslant 20 \%$ in the sum of the longest diameter of all target lesions, or the appearance of one or more new lesions and/or unequivocal progression of existing, nontarget lesions. Stable disease (SD) was defined as neither sufficient lesion shrinkage to qualify for a PR, nor sufficient increase to qualify for PD. Best response was defined as the most $\mathrm{CR}$ achieved by a patient (thus, each patient had a single best response: $C R, P R, S D$ or $P D)$, and the date of best response was the date it was first detected. Time to progression (TTP) was defined as the time from the first medication to the date of a PD event or death (due to endometrial cancer or study drugs). All tumours were radiographically assessed for response every 6 weeks. An independent response review committee (IRRC) evaluated all tumour responses after the investigators had completed their judgement.

Toxicities were evaluated with respect to incidence and severity using National Cancer Institute common toxicity criteria (NCICTC, version 2.0) (Trotti et al, 2000).

\section{Statistical consideration}

Assuming a response rate of $20 \%$, the study was designed with $80 \%$ power such that the lower limit of the $95 \%$ confidence interval (CI) for the estimate of the response rate was greater than 0.05 . A sample size of 32 evaluable patients was required.

The primary end point was overall tumour response (determined by the IRRC) with the corresponding 95\% CI using the exact binominal method for the evaluable population. The secondary end point of this study was safety. The Kaplan-Meier (KM) method was used to determine the TTP and median survival time (MST) in the evaluable population.

\section{RESULTS}

\section{Patient characteristics}

A total of 33 patients were enrolled on the study from April 2001 to October 2003 and one patient was unevaluable as a result of having received prior treatment with paclitaxel and doxorubicin-platinum regimens. The median age of the intent to treat (ITT) population ( $n=33$ ) was 59 years (range $39-74)$ and $70 \%$ patients had ECOG PS 0 (Table 1). Several patients had unfavourable histologic characteristics: adenosquamous features (three) and uterine papillary serous cancers (two). Most patients (88\%) had undergone total abdominal hysterectomy and bilateral salpingooophorectomy, and one-third of patients had prior radiotherapy. Of those patients who had received prior chemotherapy $(n=14)$, 10 had received combination doxorubicin-platinum in combination, three had received platinum alone and one had received oral fluorouracil. All 33 patients were evaluated for toxicity and survival, while 32 patients were evaluated for response and TTP.

\section{Treatment delivery}

Overall, 32 patients received a total of 133 cycles of docetaxel and the median number of cycles of docetaxel was four (range, 1-13). Five patients (15\%) experienced dose reductions for the following reasons: two patients experienced febrile neutropenia (in one patient this occurred twice) and three patients had grade 3 nonhaematologic toxicities: diarrhoea (occurred twice in one patient), hyperglycaemia, hyperkalaemia and supraventricular tachycardia.

\section{Response}

Table 2 presents the assessment of response to treatment. Two patients, one who was chemotherapy-naïve and the other who had received prior therapy, were not assessable for response because they had received only one cycle of treatment. Before evaluation by the IRRC, primary physicians had reported two CRs and nine PRs. The IRRC judged one CR as a PR, two PR as SD and one SD as a PR. Therefore, the overall response rate for 10 of 32 patients was $31 \%$ (95\% CI, $16.1-50.0 \%)$. Of 13 patients who had prior chemotherapy, three (23\%) achieved a PR: two had received doxorubicin-platinum and one platinum alone. The histologic analysis revealed responses among the following tumour types: 
endometrioid adenocarcinoma (6 of 25 patients), squamous differentiated adenocarcinoma ( 1 of 3 ), papillary serous ( 2 of 2 ) and undifferentiated cancer ( 1 of 1 ). The median time for the onset of effect was 2.0 months (range, $0.7-4.5$ ) and the median duration of response was 1.8 months (range, 0.9-4.6). The median followup time was 17.6 months (range, 1.7-36.3) and median TTP was 3.9 months (95\% CI, 1.5-10.2 months) (Figure 1). Median survival time was 17.8 months (95\% CI, 7.4-22.0 months).

\section{Safety and toxicity}

In all, 33 patients were assessable for toxicity (Table 3). Also, 31 (94\%) patients experienced grade 3 or 4 neutropenia, and three

Table I Patient characteristics

\begin{tabular}{|c|c|}
\hline Characteristic & No. of patients $(n=33)$ \\
\hline \multicolumn{2}{|l|}{ Age, years } \\
\hline Median & 59 \\
\hline Range & $39-74$ \\
\hline \multicolumn{2}{|l|}{ ECOG performance status } \\
\hline 0 & 23 \\
\hline I & 9 \\
\hline 2 & I \\
\hline \multicolumn{2}{|l|}{ Disease status } \\
\hline Stage III, IV & 9 \\
\hline Recurrent & 24 \\
\hline \multicolumn{2}{|l|}{ Histology } \\
\hline Endometrioid & 26 \\
\hline Adenocarcinoma with squamous differentiated & 3 \\
\hline Papillary serous & 2 \\
\hline Adenocarcinoma, unspecified & 1 \\
\hline Undifferentiated & I \\
\hline \multicolumn{2}{|l|}{ Tumour grade } \\
\hline 1 & 11 \\
\hline 2 & $\mid 1$ \\
\hline 3 & 6 \\
\hline Unknown & 5 \\
\hline \multicolumn{2}{|l|}{ Prior treatment } \\
\hline Surgery & 29 \\
\hline Radiotherapy & 9 \\
\hline Hormonal therapy & 5 \\
\hline \multicolumn{2}{|l|}{ Prior chemotherapy } \\
\hline None & 19 \\
\hline Doxorubicin and platinum & 9 \\
\hline Platinum alone & 3 \\
\hline Others & 2 \\
\hline
\end{tabular}

ECOG $=$ Eastern Cooperative Oncology Group.
(9\%) developed febrile neutropenia. Nonhaematologic toxicities included grade 3 anorexia and vomiting experienced by some patients (18 and $9 \%$, respectively). One patient experienced grade 3 peripheral neuropathy (sensory and motor) after five treatment cycles. Three patients terminated the study as a consequence of the following toxicities: infection with Mycobacterium avium complex (one), grade 4 hypersensitivity reaction despite premedication with dexamethasone (one) and grade 3 oedema with pleural effusion after six treatment cycles (one). All three patients recovered after receiving recommended medical treatment. There were no treatment-related deaths.

\section{DISCUSSION}

At initial diagnosis, only a small percentage of endometrial cancer patients have recurrent or advanced disease with distant metastases, and therefore a multicentre trial is essential for the accrual of patients. This multicentre phase II trial, although relatively small in sample size, clearly demonstrated that docetaxel is active in the treatment of endometrial cancer. Toxicity was manageable and predominantly haematologic.

Taxanes have shown activity in this setting previously, with paclitaxel demonstrating overall response rates of $27-37 \%$ when used as a single agent in endometrial cancer (Ball et al, 1996; Lissoni et al, 1996; Lincoln et al, 2003). Combination chemotherapy with paclitaxel and carboplatin or cisplatin has resulted in response rates of 50-56\% (Dimopoulos et al, 2000; Hoskins et al,

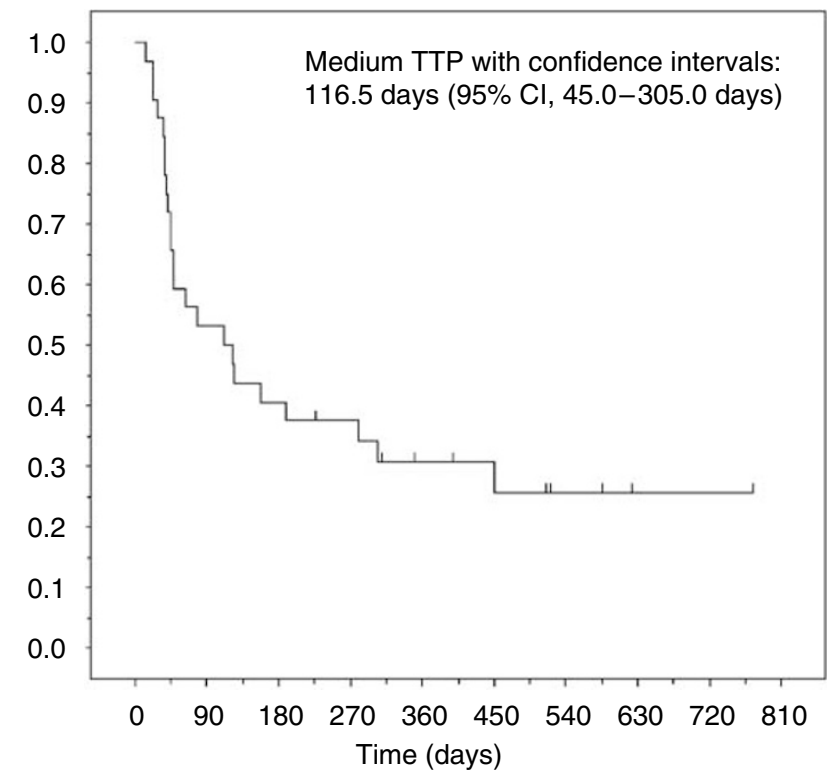

Figure I KM curve of estimated TTP.

Table 2 Best response (RECIST criteria) to docetaxel

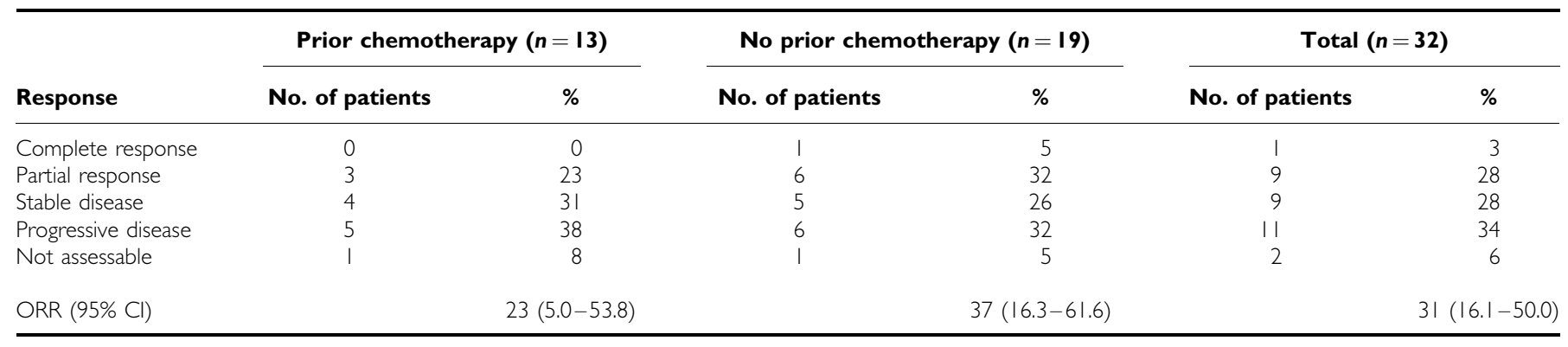

$\mathrm{ORR}=$ overall response rate; $\mathrm{Cl}=$ confidence interval. 


\begin{tabular}{|c|c|c|c|c|c|c|c|c|c|c|}
\hline \multirow[b]{3}{*}{ Toxicities } & \multicolumn{10}{|c|}{ NCI-CTC grade $(n=33)$} \\
\hline & \multicolumn{2}{|c|}{ I } & \multicolumn{2}{|c|}{2} & \multicolumn{2}{|c|}{3} & \multicolumn{2}{|c|}{4} & \multicolumn{2}{|c|}{$3-4$} \\
\hline & No. & $\%$ & No. & $\%$ & No. & $\%$ & No. & $\%$ & No. & $\%$ \\
\hline Neutrophils & I & 3 & 0 & 0 & 10 & 30 & 21 & 64 & 31 & 94 \\
\hline Haemoglobin & | | & 33 & || & 33 & 1 & 3 & I & 3 & 2 & 6 \\
\hline Lymphopenia & 1 & 3 & 14 & 42 & $\mid 1$ & 33 & - & || & 33 & \\
\hline Platelets & 6 & 18 & 1 & 3 & 0 & 0 & 0 & 0 & 0 & 0 \\
\hline Alopecia & 5 & 15 & 26 & 79 & - & - & - & & & \\
\hline Fatigue & 13 & 39 & 7 & 21 & 3 & 9 & 0 & 0 & 3 & 9 \\
\hline Anorexia & 12 & 36 & 5 & 15 & 6 & 18 & 0 & 0 & 6 & 18 \\
\hline Nausea & 16 & 49 & 6 & 18 & 2 & 6 & - & 2 & 6 & \\
\hline Vomiting & 7 & 21 & 3 & 9 & 3 & 9 & 0 & 0 & 3 & 9 \\
\hline Diarrhoea & 14 & 42 & 3 & 9 & 3 & 9 & 0 & 0 & 3 & 9 \\
\hline Constipation & 2 & 6 & 10 & 30 & 4 & 12 & 0 & 0 & 4 & 12 \\
\hline Stomatitis & 3 & 9 & 5 & 15 & 1 & 3 & 0 & 0 & 1 & 3 \\
\hline Febrile neutropenia & - & - & 3 & 9 & 0 & 0 & 3 & 9 & & \\
\hline Infection & 0 & 0 & 3 & 9 & 0 & 0 & 0 & 0 & 0 & 0 \\
\hline Oedema & 7 & 21 & 3 & 9 & I & 3 & 0 & 0 & 1 & 3 \\
\hline Neuropathy-motor & I & 3 & 0 & 0 & I & 3 & 0 & 0 & । & 3 \\
\hline Neuropathy-sensory & 9 & 27 & 2 & 6 & I & 3 & 0 & 0 & 1 & 3 \\
\hline Supraventricular arrhythmia & 0 & 0 & 0 & 0 & I & 3 & 0 & 0 & I & 3 \\
\hline Allergic reaction & 3 & 9 & 0 & 0 & 0 & 0 & I & 3 & I & 3 \\
\hline Rash/desquamation & 6 & 18 & 5 & 15 & I & 3 & 0 & 0 & I & 3 \\
\hline Injection site reaction & 5 & 15 & 2 & 6 & 0 & 0 & 0 & 0 & 0 & 0 \\
\hline Nail changes & 4 & 12 & 0 & 0 & - & - & - & & & \\
\hline AST & 9 & 27 & 3 & 9 & 0 & 0 & 0 & 0 & 0 & 0 \\
\hline $\mathrm{ALT}$ & 8 & 24 & 2 & 6 & 0 & 0 & 0 & 0 & 0 & 0 \\
\hline Hypokalaemia & 0 & 0 & - & 3 & 9 & 0 & 0 & 3 & 9 & \\
\hline
\end{tabular}

$\mathrm{NCI}-\mathrm{CTC}=$ National Cancer Institute common toxicity criteria; AST = asparate aminotransferase; ALT=alanine aminotransferase. Present NCl-CTC grade $3-4$ in $>5 \%$ patients and breakdown if possible by whether patient had prior chemotherapy.

2001; Scudder et al, 2005). However, a GOG randomised trial of women with advanced or recurrent endometrial carcinoma, in which the combination paclitaxel-doxorubicin was compared with doxorubicin-cisplatin, showed that the paclitaxel arm did not result in an improved outcome (Fleming et al, 2000). A subsequent GOG study, in which the combination paclitaxel, doxorubicin and cisplatin (TAP) with G-CSF was compared with doxorubicin-cisplatin, showed that the TAP arm yielded a better response (57 vs 34\%; $P<0.01$ ), progression-free survival (median, 8.3 vs 5.3 months; $P<0.01$ ) and $O S$ (median, 15.3 vs 12.3 months; $P=0.037$ ) than the control arm. However, more grade 3 neuropathy (12 vs $1 \%$ ) and congestive heart failure were observed with TAP than with doxorubicin-cisplatin (Fleming et al, 2004). In light of this imbalance between efficacy and toxicity, TAP has not been accepted as the standard chemotherapy regimen in routine clinical practice.

Docetaxel has a toxicity profile that is different from paclitaxel. In particular, neurotoxicity occurs at a low incidence with docetaxel. In our study, only one patient developed grade 3 neuropathy-sensory and recovered in several weeks. While fluid retention is a distinctive toxicity of docetaxel, this can be prevented using premedication (Piccart et al, 1997); in our trial, one patient developed pleural effusion since the routine premedication with corticosteroids was not applied.

Several studies have reported on second-line chemotherapy for endometrial cancer. Two phase II trials of second-line paclitaxel report response rates of $27 \%$ (12 out of 44 ) and $37 \%$ (7 out of 19)
(Lissoni et al, 1996; Lincoln et al, 2003). An older report describes a $30 \%$ response rate to second-line high-dose cisplatin $\left(3 \mathrm{mg} \mathrm{kg}^{-1}\right)$ among 13 patients (Deppe et al, 1980). With the exception of these studies, response rates to second-line chemotherapy are uniformly less than $20 \%$ and most are less than $10 \%$ (Slayton et al, 1982, 1988; Stehman et al, 1983; Thigpen et al, 1984b, 1986; Homesley et al, 1986; Asbury et al, 1990; Muss et al, 1991, 1993; Sutton et al, 1994; Rose et al, 1996; Muggia et al, 2002). In our study, $23 \%$ of pretreated patients ( 3 out of 13 ) had a PR to docetaxel, suggesting that it too is active as second-line therapy.

In conclusion, this multicentre phase II trial shows that docetaxel is active in the treatment of chemotherapy-naïve and chemotherapy pretreated patients with advanced or recurrent endometrial cancer and possesses a manageable toxicity profile; however, the effect was transient and accompanied by pronounced neutropenia in most patients. The exploration of the efficacy of docetaxel combinations in phase III studies for the treatment of endometrial cancer is of great interest and will be initiated.

\section{ACKNOWLEDGEMENTS}

The present study was supported by an entrusted fund from Aventis Phama Ltd, Tokyo, Japan, which provided docetaxel. This trial was authorised by the institutional review boards of each participating institute.

\section{REFERENCES}

Asbury RF, Blessing JA, McGuire WP, Hanjani P, Mortel R (1990) Aminothiadiazole (NSC 4728) in patients with advanced carcinoma of the endometrium. A phase II study of the Gynecologic Oncology group. Am J Clin Oncol 13: 39-41 
Ball HG, Blessing JA, Lentz SS, Mutch DG (1996) A phase II trial of paclitaxel in patients with advanced or recurrent adenocarcinoma of the endometrium: a Gynecologic Oncology Group study. Gynecol Oncol 62: $278-281$

Bissery MC, Nohynek G, Sanderink GJ, Lavelle F (1995) Docetaxel (Taxotere): a review of preclinical and clinical experience. Part I: Preclinical experience. Anticancer Drugs 6: 339-355, 363-368

Deppe G, Cohen CJ, Bruckner HW (1980) Treatment of advanced endometrial adenocarcinoma with cis-dichlorodiammine platinum (II) after intensive prior therapy. Gynecol Oncol 10: $51-54$

Dimopoulos MA, Papadimitriou CA, Georgoulias V, Moulopoulos LA, Aravantinos G, Gika D, Karpathios S, Stamatelopoulos S (2000) Paclitaxel and cisplatin in advanced or recurrent carcinoma of the endometrium: long-term results of a phase II multicenter study. Gynecol Oncol 78: $52-57$

Fleming GF, Brunetto VL, Bentley R, Rader J, Clarke-Pearson D, Asorosky J, Eaton L, Gallion H, Gibbons WE (2000) Randomized trial of doxorubicin plus cisplatin $v s$ doxorubicin plus paclitaxel plus granulocyte colonystimulating factor in patients with advanced or recurrent endometrial cancer: a report on Gynecologic Oncology Group protocol. Proc Am Soc Clin Oncol 19: 1498 (abstract)

Fleming GF, Brunetto VL, Cella D, Look KY, Reid GC, Munkarah AR, Kline R, Burger RA, Goodman A, Burks RT (2004) Phase III trial of doxorubicin plus cisplatin with or without paclitaxel plus filgrastim in advanced endometrial carcinoma: a Gynecologic Oncology Group Study. J Clin Oncol 22: 2159-2166

Homesley HD, Blessing JA, Conroy J, Hatch K, DiSaia PJ, Twiggs LB (1986) ICRF-159 (razoxane) in patients with advanced adenocarcinoma of the endometrium. A Gynecologic Oncology Group Study. Am J Clin Oncol 9: $15-17$

Hoskins PJ, Swenerton KD, Pike JA, Wong F, Lim P, Acquino-Parsons C, Lee N (2001) Paclitaxel and carboplatin, alone or with irradiation, in advanced or recurrent endometrial cancer: a phase II study. J Clin Oncol 19: $4048-4053$

Hsu Y, Sood AK, Sorosky JI (2004) Docetaxel vs paclitaxel for adjuvant treatment of ovarian cancer. Case - control analysis of toxicity. Am J Clin Oncol 27: 14-18

Lincoln S, Blessing JA, Lee RB, Rocereto TF (2003) Activity of paclitaxel as second-line chemotherapy in endometrial carcinoma: a Gynecologic Oncology Group study. Gynecol Oncol 88: 277-281

Lissoni A, Zanetta G, Losa G, Gabriele A, Parma G, Mangioni C (1996) Phase II study of paclitaxel as salvage treatment in advanced endometrial cancer. Ann Oncol 7: 861-863

Moore TD, Phillips PH, Nerenstone SR, Cheson BD (1991) Systemic treatment of advanced and recurrent endometrial carcinoma: current status and future directions. J Clin Oncol 9: 1071-1088

Muggia FM, Blessing JA, Sorosky J, Reid GC (2002) Phase II trial of the pegylated liposomal doxorubicin in previously treated metastatic endometrial cancer: a Gynecologic Oncology Group Study. J Clin Oncol 20: $2360-2364$

Muss HB, Blessing JA, DuBeshter B (1993) Echinomycin in recurrent and metastatic endometrial carcinoma. A phase II trial of the Gynecologic Oncology Group. Am J Clin Oncol 16: $492-493$

Muss HB, Bundy BN, Adcock L (1991) Teniposide (VM-26) in patients with advanced endometrial carcinoma. A phase II trial of the Gynecologic Oncology Group. Am J Clin Oncol 14: 36-37

Piccart MJ, Klijn J, Paridaens R, Nooij M, Mauriac L, Coleman R, Bontenbal M, Awada A, Selleslags J, Van Vreckem A, Van Glabbeke M (1997) Corticosteroids significantly delay the onset of docetaxel-induced fluid retention: final results of a randomized study of the European Organization for Research and Treatment of Cancer Investigational Drug Branch for Breast Cancer. J Clin Oncol 15: 3149-3155

\section{Appendix}

The following institutions (with principal investigators) participated in this study: Sapporo Medical University, Sapporo, Satoru Sagae; Niigata University, Niigata, Kenichi Tanaka; Tochigi National Hospital, Utsunomiya, Masaaki Kikuchi; National hospital Organization Saitama Hospital, Wako, Mikio Mikami; National Cancer Center Hospital, Tokyo, Noriyuki Katsumata; Tokyo Women's Medical University, Tokyo, Hiroaki Ohta; School of medicine, Keio University, Tokyo, Daisuke Aoki; St. Marianna University School of Medicine, Kawasaki, Kazushige Kiguchi;
Rose PG, Blessing JA, Lewandowski GS, Creasman WT, Webster KD (1996) A phase II trial of prolonged oral etoposide (VP-16) as second-line therapy for advanced and recurrent endometrial carcinoma: a Gynecologic Oncology Group Study. Gynecol Oncol 63: 101-104

Scudder SA, Liu PY, Wilczynski SP, Smith HO, Jiang C, Hallum III AV, Smith GB, Hannigan EV, Markman M, Alberts DS (2005) Paclitaxel and carboplatin with amifostine in advanced, recurrent, or refractory endometrial adenocarcinoma: a phase II study of Southwest Oncology Group. Gynecol Oncol 96: 610-615

Slayton RE, Blessing JA, Delgado G (1982) Phase II trial of etoposide in the management of advanced or recurrent endometrial carcinoma: a Gynecologic Oncology Group Study. Cancer Treat Rep 66: $1669-1671$

Slayton RE, Blessing JA, DiSaia PJ, Phillips G (1988) A phase II clinical trial of diaziquone in the treatment of patients with recurrent endometrial carcinoma. A Gynecologic Oncology Group study. Am J Clin Oncol 11: $612-613$

Stehman FB, Blessing JA, Delgado G, Louka M (1983) Phase II evaluation of dianhydrogalactitol in the treatment of advanced endometrial adenocarcinoma: a Gynecologic Oncology Group Study. Cancer Treat Rep 67: $737-738$

Sutton GP, Blessing JA, Homesley HD, McGuire WP, Adcock L (1994) Phase II study of ifosfamide and mesna in refractory adenocarcinoma of the endometrium. A Gynecologic Oncology Group study. Cancer 73: $1453-1455$

Therasse P, Arbuck SG, Eisenhauer EA, Wanders J, Kaplan RS, Rubinstein L, Verweij J, Van Glabbeke M, van Oosterom AT, Christian MC, Gwyther SG (2000) New guidelines to evaluate the response to treatment in solid tumors. European Organization for Research and Treatment of Cancer, National Cancer Institute of the United States, National Cancer Institute of Canada. J Natl Cancer Inst 92: 205-216

Thigpen JT, Blessing JA, Homesley H, Creasman WT, Sutton G (1989) Phase II trial of cisplatin as first-line chemotherapy in patients with advanced or recurrent endometrial carcinoma: a Gynecologic Oncology Group Study. Gynecol Oncol 33: 68-70

Thigpen JT, Blessing JA, Homesley HD, Petty W (1986) Phase II trial of piperazinedione in the treatment of advanced or recurrent endometrial carcinoma. A Gynecologic Oncology Group Study. Am J Clin Oncol 9: $21-23$

Thigpen JT, Blessing JA, Lagasse LD, DiSaia PJ, Homesley HD (1984a) Phase II trial of cisplatin as second-line chemotherapy in patients with advanced or recurrent endometrial carcinoma. A Gynecologic Oncology Group study. Am J Clin Oncol 7: 253-256

Thigpen JT, Blessing JA, Lagasse LD, DiSaia PJ, Homesley HD (1984b) Phase II trial of cisplatin as second-line chemotherapy in patients with advanced or recurrent endometrial carcinoma. A Gynecologic Oncology Group study. Am J Clin Oncol 7: 253-256

Thigpen JT, Brady MF, Homesley HD, Malfetano J, DuBeshter B, Burger RA, Liao S (2004) Phase III trial of doxorubicin with or without cisplatin in advanced endometrial carcinoma: a Gynecologic Oncology Group Study. J Clin Oncol 22: 3902-3908

Trotti A, Byhardt R, Stetz J, Gwede C, Corn B, Fu K, Gunderson L, McCormick B, Morrisintegral M, Rich T, Shipley W, Curran W (2000) Common toxicity criteria: version 2.0. An improved reference for grading the acute effects of cancer treatment: impact on radiotherapy. Int J Radiat Oncol Biol Phys 47: 13-47

van Wijk FH, Lhomme C, Bolis G, Scotto di Palumbo V, Tumolo S, Nooij M, de Oliveira CF, Vermorken JB (2003) Phase II study of carboplatin in patients with advanced or recurrent endometrial carcinoma. A trial of the EORTC Gynaecological Cancer Group. Eur J Cancer 39: 78-85

Kitasato University, Sagamihara, Hiroyuki Kuramoto; Nagoya City University, Nagoya, Atsushi Arakawa; Fujita Health University, Toyoake, Yasuhiro Udagawa; Aichi Cancer Center Hospital, Nagoya, Kazuo Kuzuya; Osaka Medical College, Takatsuki, Ken Ueki; Kinki University, Osakasayama, Hiroshi Hoshiai; Hyogo Medical Center for Adults, Akashi, Ryuichiro Nishimura; Wakayama Medical University, Wakayama, Katsuji Kokawa; Okayama University, Okayama, Junichi Kodama; Okayama Red Cross General Hospital, Okayama, Kohei Ejiri; Kawasaki Medical School, Kurashiki, Ichiro Kohno; National Kyushu Cancer Center, 
Fukuoka, Toshiaki Saito; Kyusyu University, Fukuoka, Toshio Hirakawa; Fukuoka University, Fukuoka, Toru Hachisuga; University of Occupational and Environmental Health, Kitakyuushu, Naoyuki Toki; Kurume University, Kurume, Toshiharu Kamura;

Kurume University Medical Center, Kurume, Naofumi Okura; Aso Iizuka Hospital, Iizuka, Yasuhito Sohda; Saga University, Saga, Tsuyoshi Iwasaka; Kagoshima City Hospital, Kagoshima, Masayuki Hatae; Tokyo Teishin Hospital, Tokyo, Hiroki Hata. 